\title{
Electrostatic Field Calculations for Liquid Nitrogen Gaps Assuming a Decisive Field Factor
}

\author{
Stefan Fink \\ Karlsruhe Institute of Technology (KIT), ITEP, Germany
}

\begin{tabular}{l}
\hline \hline Article Info \\
\hline Article history: \\
Received Sep 18, 2017 \\
Revised Feb 23, 2018 \\
Accepted Mar 7, 2018 \\
\hline
\end{tabular}

Keyword:

Breakdown voltage

Liquid nitrogen

\begin{abstract}
Volume effect on breakdown voltage is well known in high voltage engineering. The breakdown voltage behavior of liquid nitrogen depending on a high field volume had been quantitatively described for gap lengths up to $20 \mathrm{~mm}$. Breakdown curves for longer gap lengths up to $96 \mathrm{~mm}$ derived from measurements with a facility "Fatelini 2" show oscillations and partly low withstand voltages. Electrostatic field calculation for such long gaps shows remarkable high field volume differences between a model for ideal sphere and models including fixation rods. Calculation for the used setup does not show monotonically increasing high field volume depending on gap length but a maximum around $60 \mathrm{~mm}$ which can explain the special breakdown behavior in a "mid range" gap length. Further high field calculations were done for not yet used setups in order to make considerations, e.g. for the influence of cryostat material or diameter.
\end{abstract}

Copyright $@ 2018$ Institute of Advanced Engineering and Science. All rights reserved.

\section{Corresponding Author:}

Stefan Fink,

KIT-Campus Nord-Institut für Technische Physik,

Hermann-von-Helmholtz-Platz 1, 76344 Eggenstein-Leopoldshafen, Germany.

Email: fink@kit.edu

\section{INTRODUCTION}

Present superconducting apparatus for power engineering like fault current limiters [1] or power transmission cables [2] are usually based on high temperature superconductors with liquid nitrogen as cooling material. The determination of the dielectric strength is necessary in case of using the liquid nitrogen also for high voltage insulation purposes. Breakdown behavior of liquid nitrogen is investigated by numerous researchers but for gap lengths of more than $20 \mathrm{~mm}$ it is difficult to find experimental data [3-5].

High voltage tests with a "sphere" to plane setup with gaps up to $35 \mathrm{~mm}$ for negative [6] and $60 \mathrm{~mm}$ for positive [6-7] standard lightning impulse as well as for AC voltage [7] and gap lengths up to $96 \mathrm{~mm}$ had been performed in steps in a facility called "Fatelini 2". The withstand voltage values had been specified as highest voltage step of 20 standard lightning impulses or $15 \mathrm{~min}$ AC voltage duration where no breakdown occurs.

A transition from the superconducting to the normal conductive state within a superconducting apparatus may cause degradation of the dielectric strength of liquid nitrogen caused by gas bubble generation. Hence a test series with bubble generation by an ohmic heater was also performed in addition to the "normal" test conditions with slightly boiling liquid nitrogen within a low loss metal cryostat equipped with a thermal liquid nitrogen shield in addition to the thermal vacuum insulation.

The negative standard lightning impulse values showed linear increase depending on the gap length up to the impulse facility limit of $365 \mathrm{kV}$. In contradiction a tendency of swinging was found for positive impulses and AC (facility limit for AC tests was $230 \mathrm{kVrms}=325 \mathrm{kVpeak}$ ). For the positive impulse and AC results (Figure 1) significant "oscillations" occur especially between $20 \mathrm{~mm}$ and $70 \mathrm{~mm}$. These oscillations are a considerable setup issue but an explanation is not given so far. Numerical calculations will be used to 
solve this problem and based on this method a prediction for the breakdown behavior of possible future setups is presented.

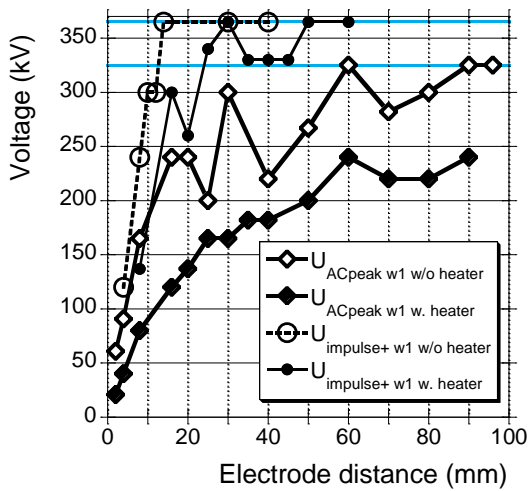

Figure 1. Withstand voltages of liquid nitrogen for a pressure of $0.1 \mathrm{MPa}$ ("w1") depending on electrode distance [7]. Peak alternating and positive standard impulse ("impulse+") voltages with $500 \mathrm{~W}$ operation of a heater ("w. heater", filled markers) and without excitation of the heater ("w/o heater", unfilled markers). The maximum available high voltage test facility values of $325 \mathrm{kV}$ for AC operation and $365 \mathrm{kV}$ for standard lightning impulse operation are indicated by blue horizontal lines

\section{RESEARCH METHOD}

The potential reduction of breakdown voltages by increasing field stressed area and volume size is a well known phenomena in high voltage engineering and it is frequently described for liquid nitrogen breakdown behavior. Not the complete liquid nitrogen test volume should be considered for determination of breakdown behavior but only the volume where the electric field exceeds a certain voltage in relation to the maximum field value. The relation between this threshold field strength and the maximum field strength is called the decisive field factor. The decisive field factor is depending on pressure and temperature. A very detailed investigation for uniform and weakly non-uniform fields for gap lengths up to $20 \mathrm{~mm}$ is performed by $\mathrm{N}$. Hayakawa in [8] and allows a selection of a decisive field factor of 0.82 for the examined test conditions of Figure 1. The maximum field value of a simple sphere to plane setup can be determined by several tools like analytical formulae or tables based on conformal mapping [9], numerical field calculation or by literature search [10]. Numerical field calculation was selected as most appropriate tool for this cryogenic application considering the need for determination of the volume "V82", where the electric field is higher than $82 \%$ of the maximum field. It should be noticed that numerical field calculation is well established for room temperature high voltage engineering, e.g. [11-12]. The software Comsol Multiphysics was used in version $4.3 \mathrm{~b}$. This program offers a sweep mode for comfortable data variation (e.g. gap length) and also the possibility to solve $3 \mathrm{D}$ problems.

\subsection{Reliability of the used field calculation tool}

The possible occurrence of mathematical problems must be taken in account by using field calculation programs. Typical problems while using electrostatic field calculation occur for the determination of the maximum field strength on sharp edges, triple points or at discontinuities. The used program offers different possibilities of field strength determination. Three methods were used: clicking in field plots, an expression tool "derived values" (which offers comprehensive possibilities of calculation of simple up to very sophisticated problems), and data export of a matrix with field values.

It can be expected that for the specified sphere to plane setup the discontinuity at the sphere surface represents the most critical issue. A comparison was done between the 3 different field determination methods for one sphere to plane geometry $(10 \mathrm{~mm}$ gap length, $50 \mathrm{~mm}$ diameter of sphere). An exported matrix of field values from a simple sphere to plane model with calculation steps of $0.01 \mathrm{~mm}$ gives an impression of the field data representation originated from Comsol (Figure 2). 


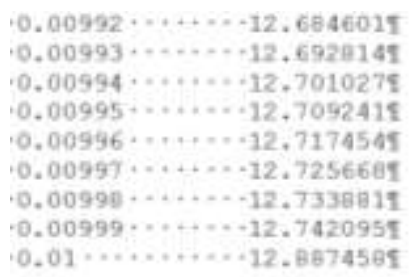

Figure 2. Example data set delivering the field strength values (second column, unit: $\mathrm{kV} / \mathrm{mm}$ ) depending on geometry variable $\mathrm{z}$ (first column, unit: $\mathrm{mm}$ ) for a simple sphere to plane model. The liquid nitrogen to steel boundary is located at $\mathrm{z}=0.01 \mathrm{~mm}$. Apparently the electric field increase of the last step is relatively high

Comsol calculates a field value even within the boundary surface itself but this value seems unexpected high if the field increase was compared with the preceding data points. In the example of Figure 2 one may expect a value of $12.75 \mathrm{kV} / \mathrm{mm}$ instead of $12.89 \mathrm{kV} / \mathrm{mm}$ which is a difference of about $1.1 \%$. In comparison the field plot clicking delivers $12.80 \mathrm{kV} / \mathrm{mm}$ and the "derived values" tool delivered $12.89 \mathrm{kV} / \mathrm{mm}$. The result of a two dimensional (2D) model of the boundary element method program CSP [13] was $12.76 \mathrm{kV} / \mathrm{mm}$.

One may now confess that the export data with following post processing or even the zoom and click method seems to deliver more accurate maximum field strength values. On the other hand the "derived value" method offers an easy to handle tool with direct calculation of the volume where the field is higher than the decisive field factor multiplied with the maximum field value. A test calculation with the derived value tool for the volume calculation of a sphere resulted in a difference of less than $1 \%$ compared to the analytical value. Finally it was decided to use the "derived value" tool for the following maximum field and volume calculations.

\subsection{Meshing}

In finite element method programs the mesh size must be adjusted in order to find a useful compromise between high solution accuracy and calculation time. Comsol offers a number of predefined mesh size qualities in order to optimize calculation time. Two sphere to plane models are shown in Figure 3.
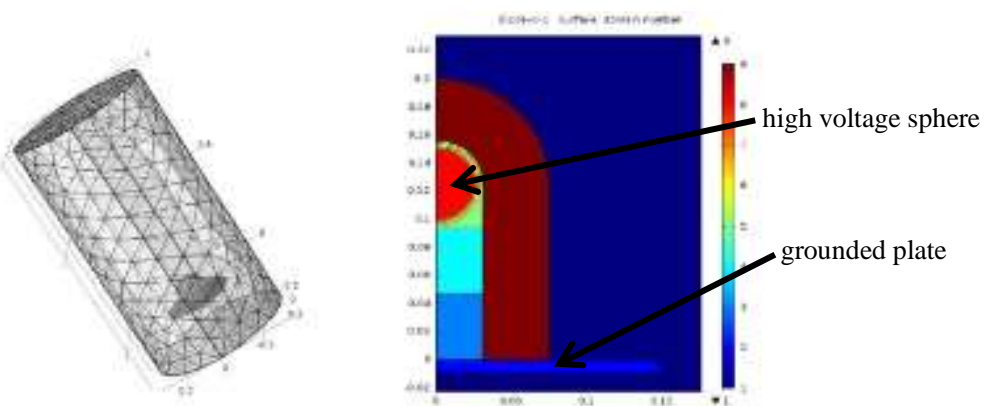

Figure 3. Full view of 3D model with coarse meshing (left) and zoomed view on a cross section of a 2D sphere to plane geometry with 9 domains (right). The innermost half circle of the 2D model represents the high voltage sphere. The plate and the cryostat walls are grounded. The other space is liquid nitrogen (i.e. 7 of the 9 domains of the 2D model; the cryostat walls are not visible in the zoomed view)

It can be useful to divide spaces (e.g. the entire liquid nitrogen space) in subspaces (Comsol: "domains") in order to improve the solution process. A further accuracy improvement can be achieved by refinement of the mesh within a box. This refinement method was used in some models for achieving high accuracy especially around the bottom point of the sphere which has the lowest distance to the plane.

Comparison of maximum field values and high field volumes for a sphere to plane setup was done between eight 2D Comsol models and five 3D Comsol models with different meshes and refinements. The most detailed 2D Comsol model was used as reference model. The simple models with only one domain for the whole liquid nitrogen space show differences of more than $1 \%$ for the maximum electric field. Maximum percentage deviation with less than $1 \%$ was found for the calculation of the maximum electrical field in case of Comsol 2D and 3D models with a liquid nitrogen space divided in several domains. A CSP model delivers a difference of $0.5 \%$ for the calculation of the maximum field value. High field volume calculation with 
maximum percentage deviation less than $1 \%$ required very detailed meshes. This had also be obtained with the most detailed 3D model but calculation time compared to the best 2D model is longer than a factor of 200 .

Hence 2D models with subdomains of the liquid nitrogen space and additional box refinement were used for the following sphere or rod to plane calculations.

\section{RESULTS AND ANALYSIS}

A theoretical 2D sphere to plane model delivered increasing high field volumes V82 with increasing gap length, e.g. a volume V82 of $1555 \mathrm{~mm}^{3}$ for $20 \mathrm{~mm}$ gap length and a volume V82 of $18674 \mathrm{~mm}^{3}$ for $100 \mathrm{~mm}$ gap length. Real world setups with fixation rods or tubes show considerable change of volume V82 for long gaps in comparison to this theoretical model although the maximum field strength is very similar (Figure 4). The Fatelini type rods with rounded half sphere design do not show a monotonically increasing behavior but have a distinct maximum. This maximum volume occurs between $50 \mathrm{~mm}$ and $70 \mathrm{~mm}$ (calculation steps are $10 \mathrm{~mm}$; the lines in Figure 4 are only for orientation). Since an increasing stressed volume causes an increasing breakdown probability these calculation results show a contribution for the explanation of the withstand voltage curve of Figure 1 between $20 \mathrm{~mm}$ and $70 \mathrm{~mm}$. It should be noticed that a diagram with V82 depending on gap length cannot be directly treated as breakdown probability curve because e. g. the maximum field value can be different for 2 points with the same volume values.
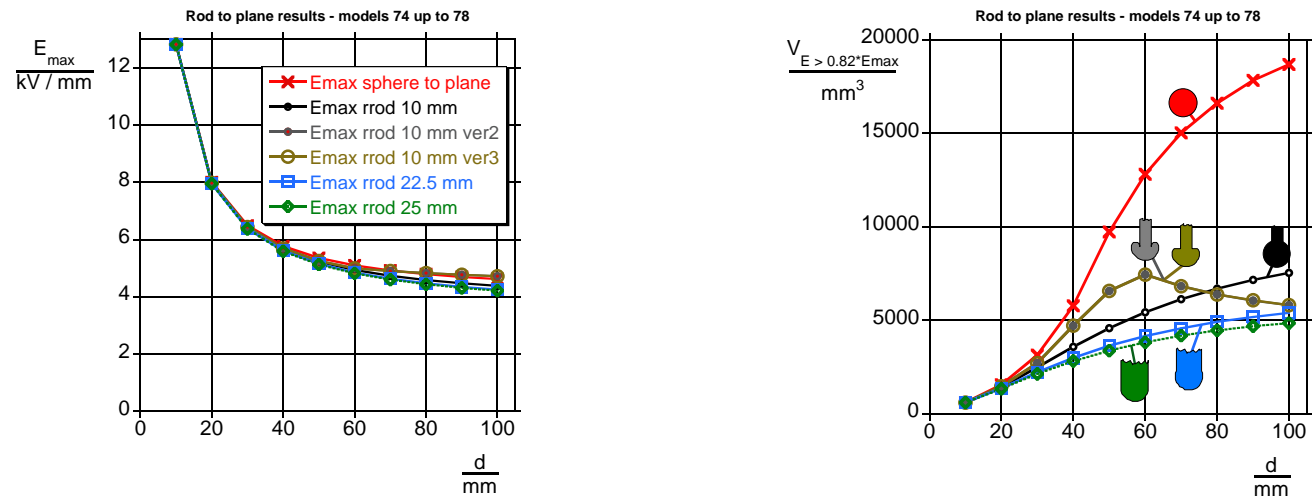

Figure 4. Maximum field Emax (left) or high stressed volume V82 (right) depending on gap length for sphere to plane and rod to plane setups. Predefined voltage is $100 \mathrm{kV}$. The rods have a $50 \mathrm{~mm}$ diameter rounding (at least $180^{\circ}$ ) termination but different radii (rrod in plot legend) of the cylindrical fixation rod

The special behavior of the rounded half spheres can be explained by comparison of field plots. A visualization for the model with $50 \mathrm{~mm}$ diameter sphere termination and rod diameter of $45 \mathrm{~mm}$ is shown in Figure 5 for 3 different gap lengths. The plate is not shown in the field plots; it is located in all plots below the sphere but also below the bottom limitation of the picture. The amount of space in the liquid nitrogen volume V82 around the bottom termination where $\mathrm{E}>0.82 *$ Emax is non white. It can be seen that even for the zoomed field plots most of the liquid nitrogen space is white which means that it does not belong to the high field region. The most "thick" color area can be found for the $10 \mathrm{~mm}$ gap with a thickness of $3.00 \mathrm{~mm}$ (at $\mathrm{r}=0$ ). For the $50 \mathrm{~mm}$ gap length the maximum thickness of V82 is $2.65 \mathrm{~mm}$ and for the $100 \mathrm{~mm}$ gap length the maximum thickness of V82 is $2.63 \mathrm{~mm}$ at r=0, each. The longer the gap length is the larger is the angle around the spherical electrode which is covered with V82 but even for the $100 \mathrm{~mm}$ gap length an angle of $90^{\circ}$ counted from the bottom point is not reached. This explains why the curve of the high field volume V82 for this model (Figure 4, blue color) is strictly monotonically increasing. 

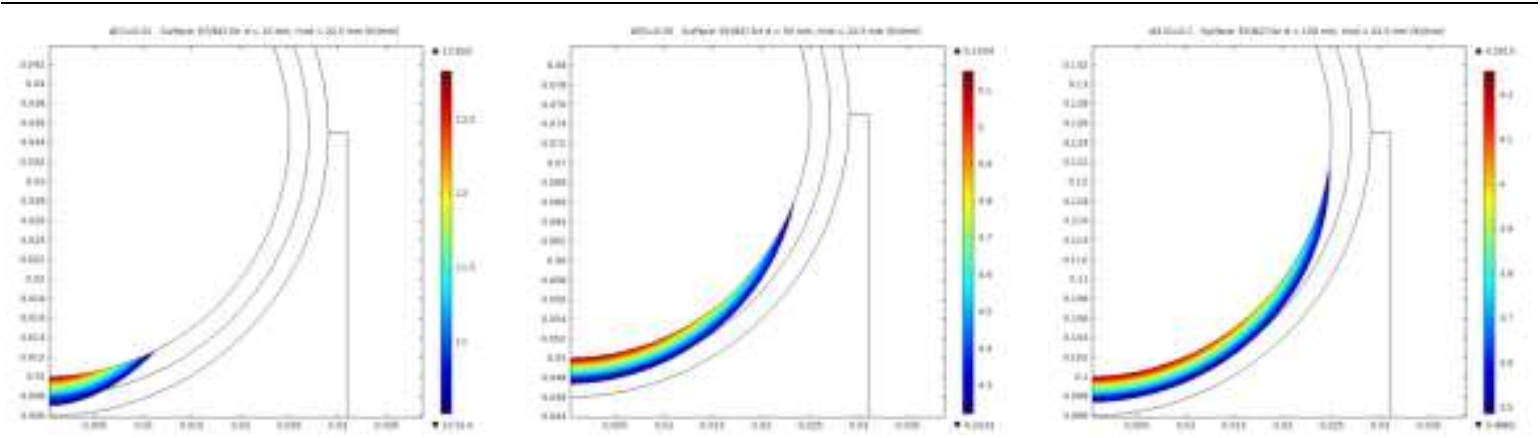

Figure 5. Plots of electric field around sphere termination (metal sphere represented by inner circle part; outer lines are only for subdomains of liquid nitrogen space) with different gap lengths of $10 \mathrm{~mm}$ (left), $50 \mathrm{~mm}$ (middle), and $100 \mathrm{~mm}$ (right) to the plane. The sphere termination diameter is $50 \mathrm{~mm}$, the rod diameter is $45 \mathrm{~mm}$. High field space with E >0.82* Emax is colored; white areas have field values not higher than $0.82 *$ Emax. Selected high voltage value is $100 \mathrm{kV}$ for the field plots, each

Figure 6 shows the V82 volume for 3 different geometries with a $20 \mathrm{~mm}$ diameter fixation rod, each, and a gap length of $100 \mathrm{~mm}$, each. The $20 \mathrm{~mm}$ diameter rod with full sphere plot (Figure 6 left, Figure 4 black curve) exceeds apparently the $90^{\circ}$ angle which explains the larger V82 volume compared to the sphere termination with the $45 \mathrm{~mm}$ diameter rod (compare Figure 5 right).

All full sphere rods have shown the maximum field stress along the point at $r=0$ which has the shortest distance to the plate. The rounded half sphere terminations show a second high field region arising in case of longer distances at the $90^{\circ}$ location. This explains the exceeding of the V82 half sphere values above the V82 sphere values for middle range distances in Figure 5. For longer distances the maximum electric field of the half sphere terminations increases to maximum values around this $90^{\circ}$ location which are even higher than the values of the artificial pure sphere model at $r=0$. This leads to a reduction of the space where the field is higher than $82 \%$ of Emax around the $r=0$ (i.e. $0^{\circ}$ ) maximum and hence the V82 volumes for the long gap lengths of both half sphere models are lower than the V82 model of the full sphere model with the same fixation rod diameter. It should be remembered that V82 diagrams are no breakdown curves. It cannot be directly concluded that an additional high field region causes breakdown probability reduction for the long gap lengths. But it can explain for Figure 1 an increase of the breakdown probability which was not given in [6] or [7] for the gap lengths above $20 \mathrm{~mm}$ up to the maximum high field volume region in Figure 4.

The following considerations describe calculations for not yet recorded setups. It can be expected that the diameter of the cryogenic test vessel (cryostat) and its material has an impact on the stressed volume V82 in case of large gaps (e.g. by creating the second high field region for the rounded half sphere gaps of Figure 6 middle and right). Figure 7 shows the results for field calculations with different metal cryostat diameter and in one case without a cryostat. The "no cryostat" case simulates the absence of metal and high permittivity material in the near environment which represents e.g. the situation of an extruded polypropylene box as simple cryostat on a wooden support and a small wire earth connection (what is not desirable from several points of view but sometimes fast practice).
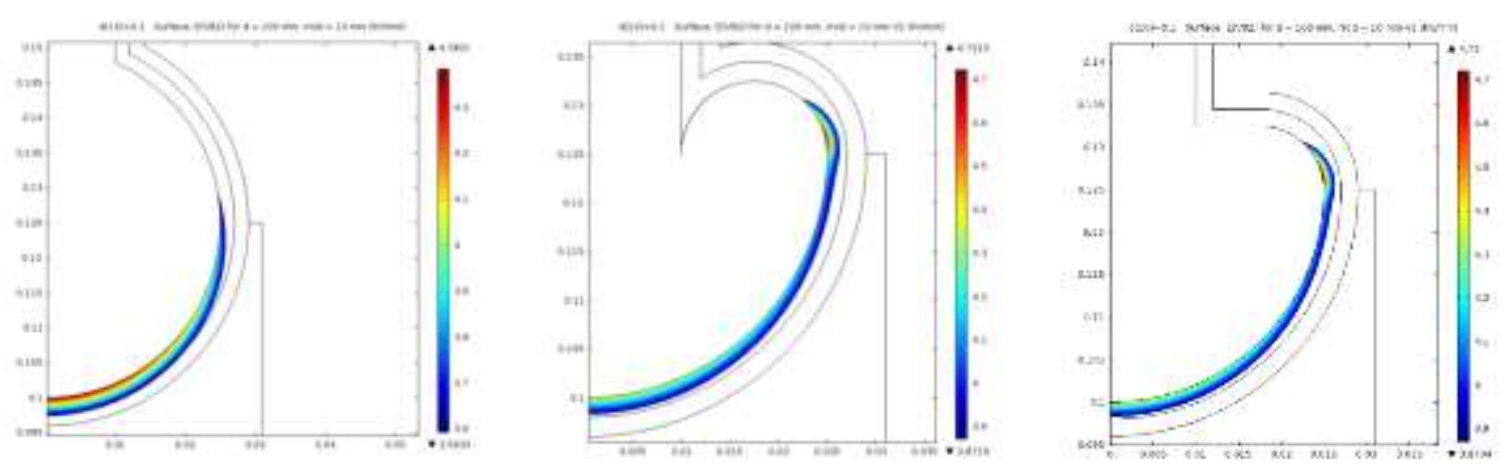

Figure 6. Field Plots E > $0.82 *$ Emax with cross section of model with $50 \mathrm{~mm}$ diameter sphere (left) or rounded half sphere (middle, right) termination and rod diameter of $20 \mathrm{~mm}$. Volume V82 is $7533 \mathrm{~mm}^{3}$ (left), $5808 \mathrm{~mm}^{3}$ (middle), and $5808 \mathrm{~mm}^{3}$ (right) 
An increasing metal cryostat diameter can have lower volume V82 for long gaps and some rod versions. Calculations for a plastic cryostat and two rod setups delivered lower volumes V82 up to a factor of about 2 compared to a metal cryostat. In case of the plastic cryostat the maximum field strength is reduced compared to a metal cryostat. One can expect for large gap experiments in plastic cryostats higher breakdown voltages compared to metal cryostats. On the other hand results obtained by tests using plastic cryostats may be very sensitive on how the grounding connection of the plate is routed and how the cryostat is installed with respect of other grounds, e. g. if the cryostat is put on a wooden desk or a grounded steel plate or near a metallic wall, etc.

The curves with the rounded half sphere model in Figure 7 (right diagram) show V82 maxima for medium gap lengths and a reversal of the larger diameter effect, e. g. for $40 \mathrm{~mm}$ gap length the smallest metal cryostat delivers highest V82 value compared to other diameters and for $80 \mathrm{~mm}$ it delivers the lowest value compared to the field values for other diameter models with the same gap length, respectively.
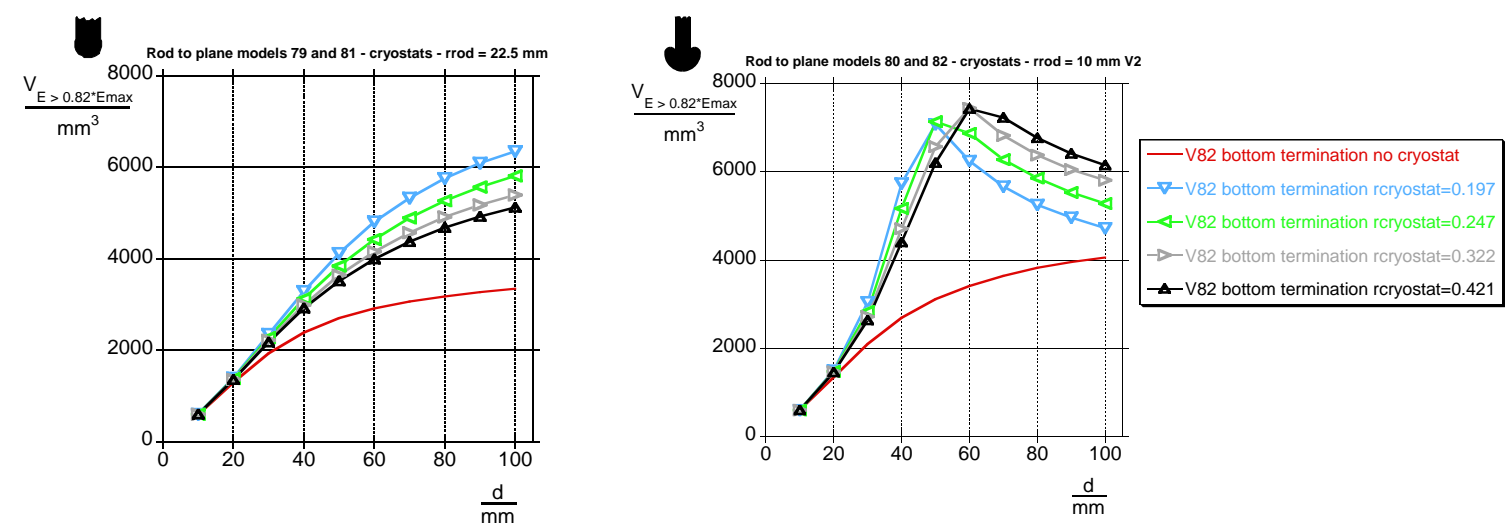

Figure 7. Calculated volume V82 depending on gap length for rod to plane setup with different cryostat radii (in $\mathrm{m}$ ) or in one case without metal cryostat walls ("no cryostat", red curve). The left picture shows a plot with a full sphere termination and a thick (diameter $45 \mathrm{~mm}$ ) fixation rod; the right picture shows a rounded half sphere termination with a thinner rod (diameter $20 \mathrm{~mm}$ )

Decisive field factors between 0.81 and 0.93 are reported in [8]. Two different geometries were examined concerning the variation of the field factor $\alpha$ for a cryostat with a stainless steel diameter of $644 \mathrm{~mm}$. Increasing decisive field factor $\alpha$ causes decreasing volumes $\mathrm{V} \alpha$. The volume maximum for the half sphere termination model appears in medium gap lengths around $60 \mathrm{~mm}$. For this model the volume of a $100 \mathrm{~mm}$ gap length and $\alpha=0.93$ was only $69 \mathrm{~mm}^{3}$ corresponding to about 1 water drop volume $\left(50 \mathrm{~mm}^{3}\right)$.

An other issue is the occurrence of a "jump" on high field volumes for a plane to plane electrode setup with edges and gap length between $10 \mathrm{~mm}$ and $20 \mathrm{~mm}$ and low decisive field factor of 0.81 . Such a "jump" can already be calculated for short gaps and slightly higher decisive field factor. This does not mean that only slight changes of gap length can have strong impact on high field volume but also a slight change of temperature or pressure can have strong impact on the calculated high field volume. As example Fig. 8 shows a fast high field volume increase for a decisive field factor change (caused e.g. by pressure decrease) from 0.84 to 0.83 . It is not experimentally examined up to now if such remarkable change of the high field volume distribution from the uniform region to the edge space is modelling reality, i.e. if a sudden reduction of breakdown voltage occurs by a related pressure loss. 

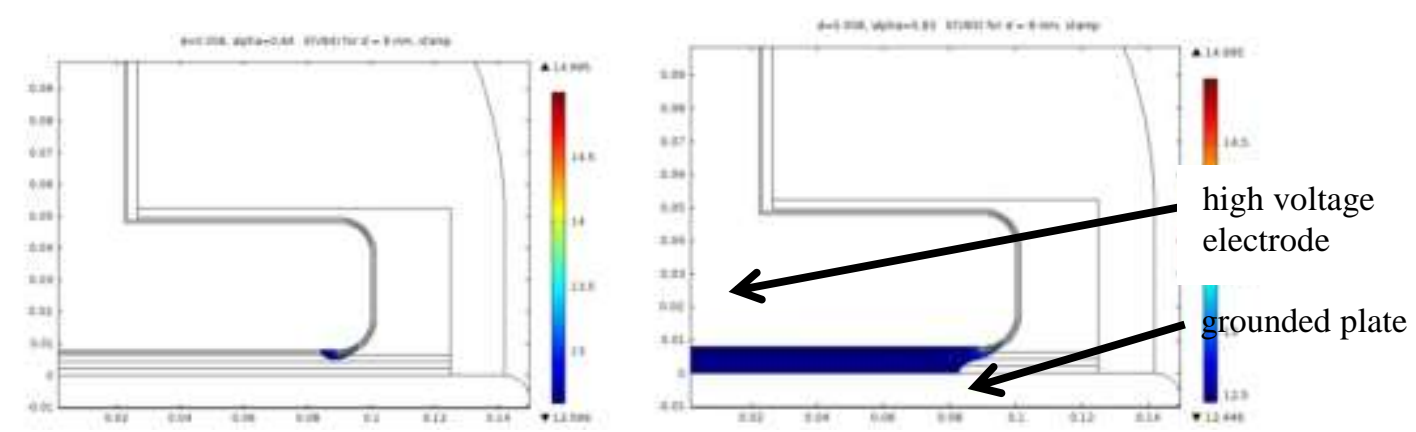

Figure 8. Field plots for a ground disk (bottom, diameter: $300 \mathrm{~mm}$ ) to high voltage "stamp" (disk with suspension rod, disk diameter: $200 \mathrm{~mm}$, edges of disk rounded) and a gap length of $8 \mathrm{~mm}$. Left plot shows high field volume for a decisive field factor of 0.84 and right figure for a decisive field factor of 0.83 . White areas show regions with field lower than decisive field factor multiplied with Emax, Each. Field values are related to a selected high voltage of $100 \mathrm{kV}$. Most of the lines within the plots are necessary for meshing of the liquid nitrogen space in order to increase accuracy of the model

\section{CONCLUSION}

According to [8] the high field volume determines the breakdown value for a liquid nitrogen setup. This high field volume can be determined by considering only the volume where the electric field strength is higher than the highest electric field strength value multiplied with the decisive electric field factor. The decisive field factor is depending on temperature and pressure and is about 0.82 for a pressure of $0.105 \mathrm{MPa}$ (absolute) and a temperature of $77.8 \mathrm{~K}$. The computation of the high field volume calculated by volume integration within the FEM program Comsol shows for several electrode setups special dependence on gap length, e.g. maxima instead of strictly monotonous increasing of the volume. A model with a simple theoretical sphere electrode is not sufficient to estimate the relevant high field volume for sphere to plane setups with a gap length longer than $20 \mathrm{~mm}$. In addition the influence of the metal cryostat diameter or material cannot be neglected for long gap setups.

Based on the calculated high field volumes it is possible to better understand the special breakdown or withstand behavior of long gap setups. A maximum of the high field volume let expect a range with reduced dielectric strength. Field plots show more than one high field region along the high voltage electrode termination and field plots show in addition changing maximum field strength locations depending on gap length. This delivers a hint for regions with larger scattering or reduction of breakdown values. Derived from the cryostat wall calculations with long gaps higher breakdown strength for plastic cryostats can be expected but with high sensitivity to grounded objects near the setup. A high decisive field factor can cause very low volumes which can explain high breakdown voltages.

In contradiction to [8] for short gaps this paper does not contain experimental based rules for the breakdown strength depending on high stressed volume. In this long gap considerations there is no relation presented between high stressed volume and breakdown voltage. The shown withstand data of Figure 1 are not sufficient to establish an experimental based rule. It should be considered that high field volume data cannot be directly taken in comparison with the (mainly) 50\% up and down method values of [8]. Even the selection of the factor 0.82 can only be considered as tentative because it relays on lower gap experiment rules with e.g. mainly lower hydrostatic pressure (a very detailed description to the setups is often not available).

Nevertheless an increasing decisive field factor reduces the high field volume but it does not change the principle behavior of the examined sphere to plane setup. This means that the principle conclusions based on the high field volume maximum for mid range gap lengths are in this special case the same.

In the future it is planned to produce more experimental data with "Fatelini 2" with other electrode shapes as sphere (or rod) to plane. The long term goal would be to establish an experimental based curve of the breakdown or withstand values depending on gap lengths but valid for longer gaps than in [8]. In addition the influence of edge effects or influence of changing locations of field maxima regions should be examined in detail. The strong influence of the experimental conditions on the high field volume value requires detailed knowledge of the test setup (cryostat diameter, distance to metal radiation shield etc.) for values from other researches. In addition a recalculation in case of different test methods is necessary which can cause some additional uncertainty. 


\section{ACKNOWLEDGEMENTS}

We acknowledge support by Deutsche Forschungsgemeinschaft and Open Access Publishing Fund of Karlsruhe Institute of Technology.

\section{REFERENCES}

[1] D. Sharma, K. B. Sahay, "Basic concepts of superconducting fault current limiter," 2016 IEEE 1st International Conference on Power Electronics, Intelligent Control and Energy Systems (ICPEICES), Delhi, 2016, pp. 1-5

[2] M. Stemmle, F. Merschel, M. Noe and A. Hobl, "Ampacity project - Worldwide first superconducting cable and fault current limiter installation in a German city center," 22nd International Conference and Exhibition on Electricity Distribution (CIRED 2013), Stockholm, 2013, pp. 1-4

[3] I. Sauers, E. Tuncer, "Strategic Dielectrics R\&D for HTS and other OE Applications," 2009 DOE Peer Review, Alexandria, VA, p. 32

[4] M. O. Pace, I. Sauers, D. R. James, E. Tuncer and G. Polizos, "Design Tool for Liquid-Nitrogen Gaps in Superconducting Apparatus," IEEE Transactions on Applied Superconductivity, vol. 21, no. 3, pp. 1441-1444, June 2011

[5] N. Hayakawa, S. Nishimachi, H. Kojima and H. Okubo, "Size effect on breakdown strength in sub-cooled liquid nitrogen for superconducting power apparatus," IEEE Transactions on Dielectrics and Electrical Insulation, vol. 22, no. 5, pp. 2565-2571, October 2015

[6] S. Fink, W.-S. Kim, M. Noe, and V. Zwecker, "Withstand impulse voltage of liquid nitrogen in the presence of gas bubbles," International Electrical Insulation Conference (INSUCON), Birmingham, Proceedings, pp. 46-51, May 2013

[7] S. Fink, R. Mueller, M. Noe, V. Zwecker and H. R. Kim, "Withstand alternating voltage of liquid nitrogen in the presence of gas bubbles," 2014 IEEE 18th International Conference on Dielectric Liquids (ICDL), Bled, 2014, pp. 1-4

[8] N. Hayakawa, S. Nishimachi, T. Mastuoka, H. Kojima, M. Hanai and H. Okubo, "Breakdown characteristics and size effect in sub-cooled liquid nitrogen," 2014 IEEE 18th International Conference on Dielectric Liquids (ICDL), Bled, 2014, pp. 1-4

[9] H. Prinz, "Hochspannungsfelder," München: R. Oldenbourg Verlag, 1969

[10] O. Laribi, "Design and optimization of a superconducting fault current limiter using numeric field calculation," bachelor thesis, Karlruhe Institute of Technology (KIT), Institute of Electric Energy Systems and High-Voltage Engineering (IEH), 2012, p. 17

[11] G. Wang, Z. Zheng, D. Huang, Z. Huang, J. Ruan, Y. Liao, "Simplification study of FE model for $1000 \mathrm{kV}$ ac transmission line insulator string voltage and grading ring surface electric field distribution calculation", TELKOMNIKA (Telecommunication Computing Electronics and Control) Indonesian Journal of Electrical Engineering, Vol.12, No.2, Feb. 2014, pp. $1188 \sim 1195$

[12] S. Zhao, Y. Zhang, Z. Chen, H. Dong, "Calculation of electric field characteristics of insulator under sandstorm condition", TELKOMNIKA (Telecommunication Computing Electronics and Control) Indonesian Journal of Electrical Engineering, Vol.12, No.2, February 2014, pp. 1169 1176

[13] J. Berrios, "The Charge Simulation Program," User's Manual, Worcester Polytechnic Institute

\section{BIOGRAPHY OF AUTHOR}

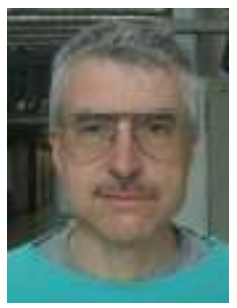

Stefan Fink finalized his apprenticeship for electrical power apparatus in 1988 and received his master degree in electrical engineering in 1994 from University of Karlsruhe, Germany. He entered the Institute for Technical Physics of KIT in 1995 and is presently leading the Cryogenic High Voltage Laboratory. His special fields of interest are high voltage engineering of cryogenic large system applications like fusion magnets, superconducting power transmission cables and fault current limiters. 